\title{
A Dynamic Frequency Allocation Scheme for IEEE 802.16 OFDMA-Based WMANs Using Hungary Algorithm
}

\author{
Shiann-Tsong Sheu, Chih-Chen Yang, and Hsu-Sheng Chang \\ Department of Communication Engineering, National Central University, Taiwain, R.O.C. \\ st.sheu@ce.ncu.edu.tw
}

\begin{abstract}
In IEEE 802.16 Wireless Metropolitan Area Networks (WMAN) with Orthogonal Frequency Division Multiple Access (OFDMA) physical layer, dynamic subchannel allocation algorithm (DSAA) is essential for a Base Station (BS) to efficiently utilize bandwidth capacity. Conventional DSAAs usually group all subcarriers into a number of subchannels and allocate subchannels to Subscriber Stations (SS), one for each SS, according to its traffic demand and channel quality of the link between BS. However, the restriction of conventional DSAAs allocating $N$ subchannels to $N$ SSs $(\mathrm{N} \geq 1)$ is inflexible and inefficient with respect to spectrum efficiency. In this paper, a new DSAA with Hungary algorithm is proposed to enhance the subchannel capacity (in bits per subcarrier) by permitting more than one subchannel allocated for one SS for which channel quality is much better than others.
\end{abstract}

Keywords: Dynamic Frequency Selection, Hungary Algorithm, Orthogonal Frequency Division Multiple Access (OFDMA), WMAN.

\section{Introduction}

The basic concept of Orthogonal Frequency Division Multiple Access (OFDMA) is to make use of OFDM modulation and allow multiple-access by dividing the whole subcarriers into a number of subchannels, which are the granular units for allocating to Subscriber Stations (SSs). Nowadays, the OFDMA system adopted by the IEEE 802.16 Mobile Wireless Metropolitan Area Network (M-WMAN) standard becomes one of the most important broadband wireless technologies.

With Orthogonal Frequency Division Multiplexing (OFDM) system, SSs use all data subcarriers of a channel to transmit data frames. However, it is hard to guarantee every SS always transmits with good channel condition. Contrarily, in OFDMA system, all data subcarriers are flexibly grouped to form a number of subchannels and one SS may be assigned to use more than one subchannel (i.e. partial subcarriers of a channel form the system viewpoint) to transmit data frames with the other SSs which are assigned to use different subchannels simultaneously. All subchannels have to be assigned to SSs in an exclusive manner. If some subchannel is assigned to improper user, the poor channel condition between BS and that user resulted from deep channel 
fading on some parts of subcarriers will degrade the transmission reliability. A smartly dynamic frequency allocation scheme should assign every subchannel to the SS which has the best channel condition with respect to that subchannel. As a result, the subchannel allocation according to the channel conditions of SSs makes SSs to transmit data frames using a more aggressive modulation and coding scheme. The issue becomes how to allocate all subchannels to SSs appropriately. Many algorithms have been proposed to resolve this issue. In paper [1], authors have proposed an iteration method to change the static allocation method to be the dynamic allocation method. The simulation results showed that it indeed increases the system capacity and decreases the bit error rate (BER) for the environments with same signal-to-noise ratio (SNR). However, the probability of making successful allocations is only $76.5 \%$ if there are 16 subchannels. In paper [2], a dynamic frequency selection is proposed to modify the method to improve the performance of the system and the successful probability of subchannel allocations. The proposed algorithm divides the subcarriers into a large number of subchannels and provides each SS one or more than one subchannel depending on the channel conditions. However, with the proposed algorithm, the number of available subchannels to be allocated to each SS must be given first. Due to this reason, one SS may be allocated to use the subchannels which might be more suitable for the other SS. So, the restriction of the given number of subchannels per SS limits the flexibility of subchannel allocations and the spectrum efficiency. Obviously, the proposed algorithm is not flexible enough and there are some shortcomings we should resolve.

To optimize the system performance, the algorithm 'Hungary Algorithm' (HA) [3] designed for the task assignment is adopted to derive the optimal subchannel assignment in this paper. We also amend HA to have the ability of flexibly assigning different numbers of subchannels to SSs according to the channel conditions of SSs

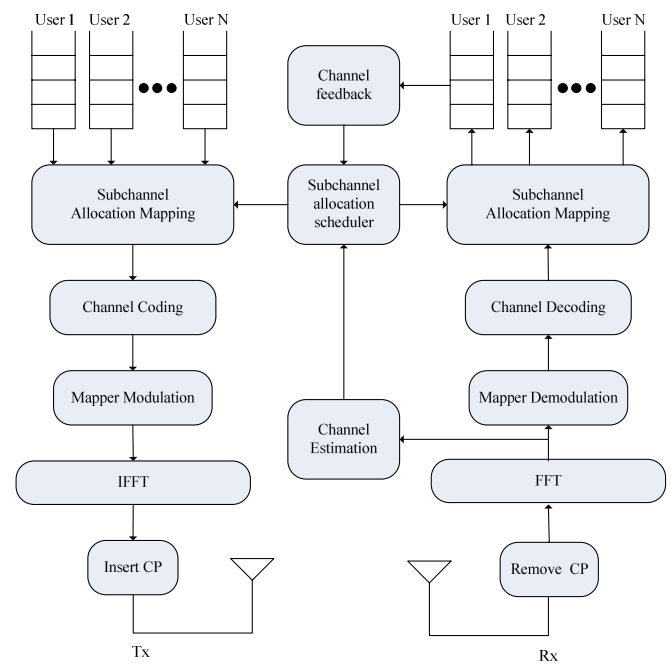

Fig. 1. System model for OFDMA system with subchannel allocation scheduler 
from time to time. Simulation results show that the proposed algorithm performs better than previous ones in terms of bit error rate (BER) under the same signal-tonoise ration (SNR) environment.

The paper is organized as follows. Section 2 shows the OFDMA system model considered in this paper. The proposed algorithm is introduced in Section 3. Simulation results and conclusions are presented in Section 4 and Section 5 respectively.

\section{System Model}

The OFDMA system block diagram of BS is showed in Figure 1. Let $\mathrm{N}$ denote the number of SSs in the system, and BS allocates data queues for SSs, one for each. Subchannel allocation scheduler is required to allocate suitable subchannels to SSs in order to increase the probability of successful frame transmissions.

As radio signal is received by the receiver of $\mathrm{BS}$, the cyclic prefix is removed first and then the signal is transformed by using Fast Fourier Transform (FFT). After then, receiver side does the channel estimation for retrieving the usefully channel condition of each subchannel to the sender SS. Next, the transformed signal is demodulated and decoded according to retrieved channel condition. Finally, subchannel allocation mapping would separate data frames from signal and put data frames to the right queues according to the subchannel allocation decision made by subchannel allocation scheduler. In data frames, there could have some channel feedback information from SSs, and such information is passed to the subchannel allocation scheduler to be the important reference for deciding subchannel allocations.

\section{Dynamic Frequency Allocation}

\subsection{Conventional Algorithms}

The simplest allocation algorithm is 'static allocation' algorithm. With this allocation algorithm, the whole subcarriers are divided into several subchannels and each subchannel is allocated to a SS by given order regardless of channel condition. However it is not an allocation algorithm to optimize the system throughput. In paper [1], the whole subcarriers are also divided into several subchannels but each subchannel is allocated to a SS who has the highest channel gain in the subchannel. By this way, the number of subchannels should be equal to the number of SSs. However, it is not a good allocation algorithm because the subchannels are assigned without considering the channel condition. Furthermore, two SSs might be chosen to use the same subchannel to transmit because of the same channel gain. For such situation, paper [2] proposes a method to solve the conflict. Let $U_{N(t-1)}$ denote the usage factor of subchannel $N$ during the previous iteration, say $t-1, C_{N}$ denote as the cost of using subchannel $N, K$ as the total number of SSs in the system and $w$ denote as the weighting factor, ranging from 0 to 1 . The usage factor of subchannel $\mathrm{N}$ during the current iteration, say $t$, is defined as follows 


$$
U_{N(t)}=U_{N(t-1)} \times w+\frac{U_{N(t-1)} \times(1-w)}{\frac{C_{N}}{(K-1)}+1} .
$$

When a subchannel is selected and allocated to more than one SS, the usage factor of that subchannel would be reduced by Eq. (1) accordingly. Contrarily, the usage factor of a subchannel that is not selected by any SS remains unchanged. However, as a consequence of subsequent normalization process, unselected subchannels will increase their usage factors whereas those selected subchannels will decrease their usage factors. In the result, the usage factor of unselected subchannel will be much close with the usage factor of selected subchannel. Furthermore, some of the SSs which select the same subchannel may choose another subchannel to transmit because the usage factor of original selected subchannel may become smaller than another usage factor of unselected subchannel. The above process is repeated until each subchannel is only allocated to one SS. After a number of iterations, the subchannels are allocated by random fashion or some forceful criteria in the case that there is still any subchannel selected by more than one SS. At most of times, the subchannel allocations are not the optimal results.

In paper [2], the paper proposed an amended method to improve the performance of the method proposed in paper [1]. The amended method divides all subcarriers into a larger number of subchannels and allocates one or more subchannels to each SS. As a result, the system performance is improved if the number of subchannels allocated to SS, which has the better channel condition, is increased. The arising probability of avoiding deep channel fading is resulted from the reduction of the number of subcarriers in each subchannel. However, the drawback of the proposed method is that the number of subchannels for an SS must be given first before the scheduling.

\subsection{Hungarian Algorithm (HA)}

The Hungarian Algorithm [3] is a famous algorithm to solve 'minimum' assignment problem with $m$ workers and $m$ jobs, and there is some limitation and pre-work before using Hungarian algorithm. The Hungarian algorithm needs to form the job assignment matrix, in which each row represents one worker, each column represents one job, and each indicates the cost if the job is assigned to the related worker. First, if the number of workers does not equal to the number of jobs, one should add dummy workers or jobs with zero costs as needed to make the matrix become $m$ by $m$ matrix. The optimal job assignment is the assignment with the minimal cost summation of all jobs assigned to workers, one for each. The steps of Hungarian algorithm are listed as follows:

Step 1 : For each row, subtract the minimum number in that row from all numbers in that row.

Step 2 : For each column, subtract the minimum number in that column from all numbers in that column.

Step 3 : Draw the minimum number of lines to cover all zeroes. If this number $=$ $m$, then STOP. An assignment can be made now.

Step 4 : Subtract $d$ (the minimum uncovered number) from uncovered numbers. Add $d$ to numbers covered by two lines. Numbers covered by one line remain the same. Then, go to Step 3. 
Then, find the minimum number of lines and determine the optimal solution.

Step 1 : Find a row or column with only one unlined zero and circle it. (If all rows/columns have two or more unlined zeroes choose an arbitrary zero.)

Step 2 : If the circle is in a row with one zero, draw a line through its column. If the circle is in a column with one zero, draw a line through its row. One approach, when all rows and columns have two or more zeroes, is to draw a line through one with the most zeroes, breaking ties arbitrarily.

Step 3 : Repeat Step 2 until all circles are lined. If this minimum number of lines equals $m$, the circles provide the optimal assignment.

Notably, if we want to solve the 'maximum' assignment problem, we need transform a the profit matrix as the needed cost matrix, in which the new cost value of every entry is equal to the absolute value of the result of original profit minus the maximal profit in profit matrix. In a word, the profit matrix is transformed to a cost matrix, and it is suitable to be solved by Hungarian algorithm.

In our system, we divide whole data subcarriers into several subchannels and use the channel information (such as RSSI, SNR, etc.) as the profit value. Considering the profit matrix $H 1$ for example, the values in matrix are assumed to be the channel conditions with respect to SSs and a larger value means a better channel condition is. Symbols $A, B, C$ and $D$ denote the four different SSs (rows) in the system, and the whole data subcarriers are divided into four subchannels (columns).

$$
H 1=\left[\begin{array}{l}
A \\
B \\
C \\
D
\end{array}\right]=\left[\begin{array}{cccc}
20 & 43 & 26 & 60 \\
42 & 84 & 6 & 9 \\
13 & 75 & 70 & 60 \\
41 & 69 & 67 & 96
\end{array}\right]
$$

Here, we want to find the subchannel assignment with the maximal profit summation from the profit matrix. As shown in matrix $H 2$, we abstract all profit values from the maximal profit first. In the result, the profit matrix is transformed to the cost matrix, and it is standard form for Hungarian algorithm to find the minimal cost solution.

$$
H 2=\left[\begin{array}{c}
A \\
B \\
C \\
D
\end{array}\right]=\left[\begin{array}{cccc}
76 & 53 & 70 & 36 \\
54 & 12 & 90 & 87 \\
83 & 21 & 26 & 36 \\
55 & 27 & 29 & 0
\end{array}\right]
$$

Next, we subtract each profit value by the minimal profit of each row and following by each column. It is shown in matrix $H 3$.

$$
H 3=\left[\begin{array}{l}
A \\
B \\
C \\
D
\end{array}\right]=\left[\begin{array}{cccc}
0 & 17 & 29 & 0 \\
2 & 0 & 73 & 75 \\
22 & 21 & 0 & 15 \\
15 & 27 & 24 & 0
\end{array}\right]
$$


As shown in matrix H3', it is fortunate that we can draw four lines to cover all zeros in the matrix.

$$
H 3^{\prime}=\left[\begin{array}{l}
A \\
B \\
C \\
D
\end{array}\right]=\left[\begin{array}{cccc}
\phi & 1 / 7 & 29 & \phi \\
2 & \phi & 73 & 75 \\
22 & 21 & 0 & 15 \\
15 & 27 & 24 & \phi
\end{array}\right]
$$

Now, we can find the optimized solution from matrix $H 3^{\prime}$ and the subchannel assignment is shown as matrix $H 4$, in which the maximal profit will be achieved if we assign the first subchannel to the SS A, the second subchannel to SS B, the third subchannel to SS C and the fourth subchannel to SS D. With this assignment, the total profit is $20+70+84+96=270$. It is indeed an optimal solution to this problem, and paper [3] has proved that the Hungarian algorithm can always find the best assignment.

$$
H 4=\left[\begin{array}{l}
A \\
B \\
C \\
D
\end{array}\right]=\left[\begin{array}{llll}
1 & 0 & 0 & 1 \\
0 & 1 & 0 & 0 \\
0 & 0 & 1 & 0 \\
0 & 0 & 0 & 1
\end{array}\right]
$$

\subsection{Enhanced Dynamic Frequency Allocation Algorithm}

Although we can get the optimal channel information by using Hungarian Algorithm, each SS just can be allocated to the pre-determined number of subchannels. In fact, while one SS suffers from bad channel condition, the SS still can get the predetermined number of subchannels. If these subchannels can be dynamically allocated to the other SSs with better channel condition, the system throughput might be increased. To meet the goal, we modify the original Hungarian Algorithm to solve the flexible subchannel assignment problem.

Here, we define a metric used for determining the maximal number of subchannels which can be allocated to each SS. The metric is defined as follows:

$$
C=\frac{P}{N} \times n
$$

where $P$ is the number of subchannels, $N$ is the number of SSs, and $n$ is the enlarging factor for providing the chance for proper $\mathrm{SS}(\mathrm{s})$ to get more subchannels. The parameter $n$ varies from $1 \mathrm{t}$ infinity and it is used to control the maximal number of subchannels which can be allocated to one SS, and $P / N$ means the average number of subchannels per SSs in this system. So, the profit matrix is extended by duplicating the channel information as needed. The problem now becomes that we have $P$ subchannels that are to be allocated to $C \times N$ SSs. For example, we divide the whole data subcarriers into four subchannels $(P=4)$ and there are four SSs $(N=4)$ in this system. When $n=2$, we have $C=2$. The profit matrix, say $H 5$, is extended to $C \times N$ by $C \times N$ matrix, in which the channel information values in entries of dummy columns are filled with 0 . Notably, it is possible that some SS get no subchannel to transmit, but no SS will use all subchannels alone. 


$$
H 5=\left[\begin{array}{l}
A_{1} \\
A_{2} \\
B_{1} \\
B_{2} \\
C_{1} \\
C_{2} \\
D_{1} \\
D_{2}
\end{array}\right]=\left[\begin{array}{cccccccc}
76 & 53 & 70 & 36 & 0 & 0 & 0 & 0 \\
76 & 53 & 70 & 36 & 0 & 0 & 0 & 0 \\
54 & 12 & 90 & 87 & 0 & 0 & 0 & 0 \\
54 & 12 & 90 & 87 & 0 & 0 & 0 & 0 \\
83 & 21 & 26 & 36 & 0 & 0 & 0 & 0 \\
83 & 21 & 26 & 36 & 0 & 0 & 0 & 0 \\
55 & 27 & 29 & 0 & 0 & 0 & 0 & 0 \\
55 & 27 & 29 & 0 & 0 & 0 & 0 & 0
\end{array}\right]
$$

After transforming the general problem to standard form, we can use Hungarian algorithm to get the matrix H6. The maximal channel gain will be achieved if we assign the first subchannel to B1, the second subchannel to B2, the third subchannel to $\mathrm{C} 1$ and the fourth subchannel to D1. With the assignment the total profit becomes $42+84+70+96=292$, and it is better than the total profit 272 derived by using original Hungarian algorithm (see matrix H4). Notably, with this assignment, SS A does not get any subchannel, SS B gets two subchannels and SSs C and D both get one subchannel. In summary, if some SS suffers from bad channel condition, it may not be assigned any subchannel. On the other hand, SS with better channel condition might get more subchannels to transmit data frames.

$$
H 6=\left[\begin{array}{l}
A_{1} \\
A_{2} \\
B_{1} \\
B_{2} \\
C_{1} \\
C_{2} \\
D_{1} \\
D_{2}
\end{array}\right]=\left[\begin{array}{llllllll}
0 & 0 & 0 & 0 & 0 & 0 & 0 & 0 \\
0 & 0 & 0 & 0 & 0 & 0 & 0 & 0 \\
1 & 1 & 0 & 0 & 0 & 0 & 0 & 0 \\
1 & 1 & 0 & 0 & 0 & 0 & 0 & 0 \\
0 & 0 & 1 & 0 & 0 & 0 & 0 & 0 \\
0 & 0 & 1 & 0 & 0 & 0 & 0 & 0 \\
0 & 0 & 0 & 1 & 0 & 0 & 0 & 0 \\
0 & 0 & 0 & 1 & 0 & 0 & 0 & 0
\end{array}\right]
$$

\section{Simulation Model and Results}

Similar to paper [1], we evaluate the effectiveness of the proposed method in the frequency selective Rayleigh channel with AWGN using Monte Carlo simulation method. We assumed that it is a four-user OFDMA system with 64 subcarriers, and these subcarriers are divided into 4 subchannels. In addition, there is no coding schemes or power control methods in the system in order to purely test the effectiveness of the proposed algorithm.

Figure 2 shows the probability of successful allocations of conventional algorithm with iteration method [1], marked as 'Iteration Algorithm', and the enhanced dynamic frequency allocation algorithm, marked as 'Hungary Algorithm'. With iteration algorithm it is possible that the subchannels can't be totally allocated to users when the number of subchannels increases. Here, we compare the probability of successful allocations between the three algorithms under different numbers of subchannels, varying from 2 to 16 . It is obvious that the probability of successful allocations is always $100 \%$ by using Hungarian algorithm. Oppositely, the probability of successful 
allocations derived from the iteration algorithm decreases as the number of subchannels increases. In the result, with Hungarian algorithm the subcarriers can be divided into more subchannels than iteration algorithms and static algorithm.

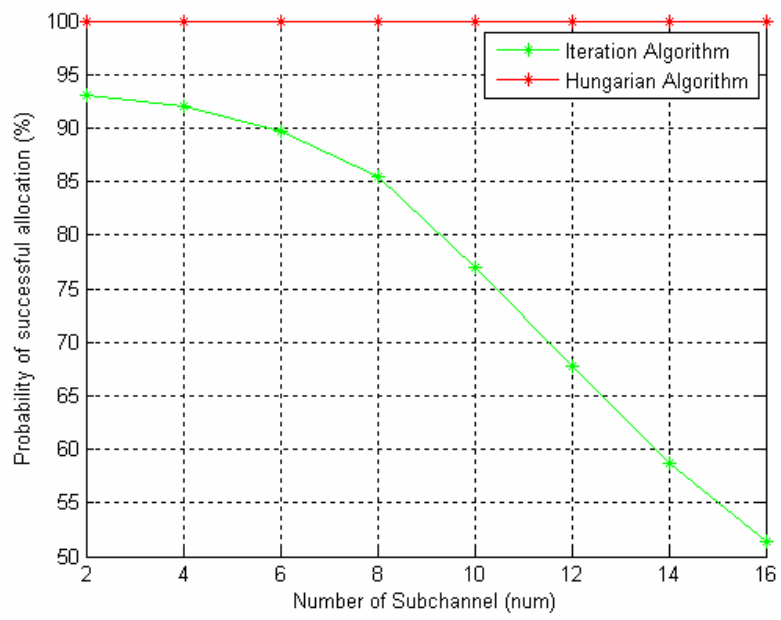

Fig. 2. The probabilities of successful allocations derived from iteration algorithm and Hungary algorithm

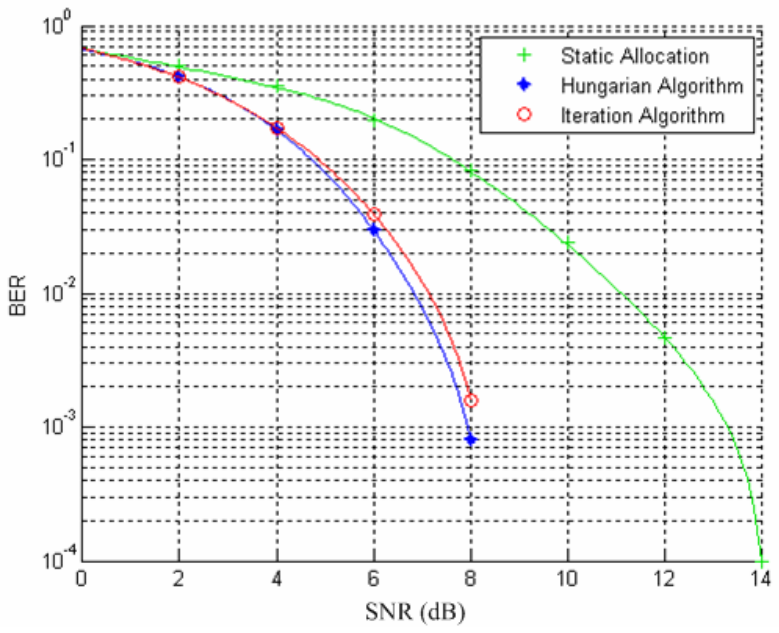

Fig. 3. Bit-Error-Rate comparisons of dynamic and static subcarrier allocation under different SNRs

The performance of subchannel allocation algorithm is the most important thing that is concerned. Figure 3 shows the performance comparisons of BERs derived from static algorithm, iteration algorithm and Hungary algorithm. From the figure, the conventionally static allocation method obtains the worst performance, and the 
performance difference among three methods becomes obvious when SNR $>4 \mathrm{~dB}$. Furthermore, not only the probability of successful allocation but also the performance with Hungarian algorithm is better than that with iteration algorithm.

Figure 4 shows the capacities of the system with static, iteration and Hungarian subchannel allocation algorithm. It can be seen that as the SNR is considered from 0 to $40 \mathrm{~dB}$, Hungarian algorithm outperforms static and iteration algorithm higher capacity under the same SNR situation. Especially, while the SNR is low the capacity of Hungarian algorithm is twice as much as the capacity of static algorithm.

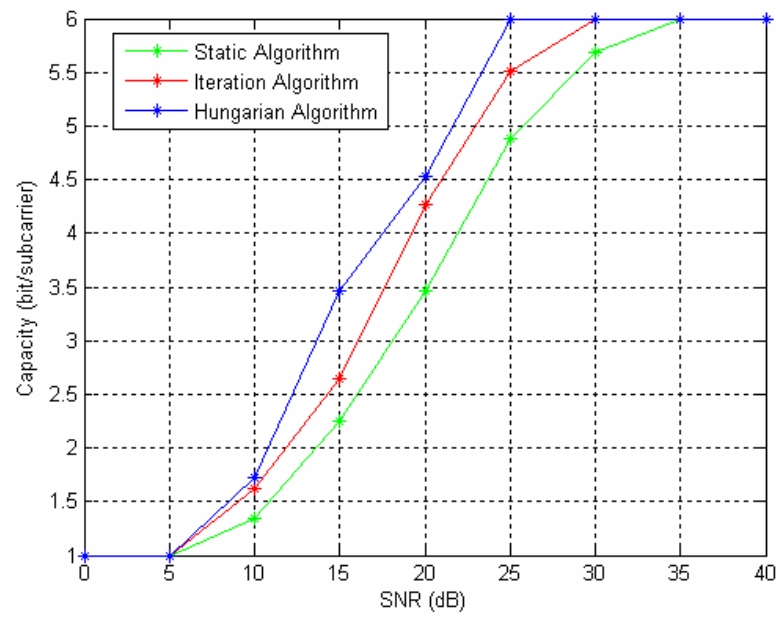

Fig. 4. Performance comparisons of dynamic and static subcarrier allocations in terms of capacity (bit/subcarrier)

\section{Conclusion}

In this paper, we proposed to use Hungarian algorithm to solve the frequency allocation problem in OFDMA system. The Hungarian algorithm is very suitable to find the optimal allocation in a short response time. However, its restriction is the number of subchannles for an SS must be given before applying Hungarian algorithm. To solve this shortcoming, an enhanced version of Hungarian algorithm is proposed to allow an SS with a better channel condition to use more subchannels than the others. Simulation results show the system throughput and BER are significantly improved by proposed methods.

\section{References}

[1] Choon, T., Alen, H., Madhukumar, A.S., Chin, F.: Capacity enhancement of a multi-user OFDM system using dynamic frequency allocation. IEEE Trans. Broadcast 49, 344-353 (2003)

[2] Chen, Y., Shon, S., Yoo, S.-J., Kim, J.M.: Dynamic frequency selection in OFDMA. In: ICACT 2006 (February 20-22, 2006) 
[3] Kuhn, H.K.: The Hungarian method for the assignment problem. Naval Research Logistics Quarterly 2, 83-97 (1955)

[4] Chong, E.K.P., Zak, S.H.: An introduction to optimization, 2nd edn. John wiley and Sons, Inc., New York (2001)

[5] Peng, Y., Doufexi, A., Armour, S., McGeehan, J.: An investigation of Dynamic sub-carrier allocation in OFDMA systems

[6] Issariyakul, T., Hossain, E.: Optimal Radio Channel Allocation for Fair Queuing in Wireless Data Networks. Parallel and Distributed Systems, IEEE Transactions 13(11), 1124-1138 (2002)

[7] Zhang, Z., He, Y., Chong, E.K.P.: Opportunistic downlink scheduling for multiuser OFDM systems. In: Wireless Communications and Networking Conference 2005, vol. 2, pp. 1206-1212. IEEE, Los Alamitos (2005)

[8] van Nee, R., Prasad, R.: OFDM for wireless multimedia communications. Artech House Publisher, Boston, London (2000) 